\title{
HOMS EFFECTS IN THE BEPC DC SEPARATOR
}

\author{
J.P. Dai, X.D. Chai, Z.T. Zhao, IHEP, Beijing, China
}

\section{Abstract}

During the operation of BEPC, the cable isolation layer near the high voltage connector of the DC separator is often melted, which is probably caused by the HOMs power. This paper presents the measurement results of the HOMs shunt impedance, the calculation of the power dissipated in the separator and through the connector, and the analysis of the HOMs effects.

\section{INTRODUCTION}

The Beijing Electron-Positron Collider (BEPC) is a high energy accelerator for hifgh energy physics and synchrotron radiation (SR) application in China. In the storage ring there are four direct current (DC) separators used to separate the electron and positron beams during the injection. BEPC usually operates in two modes: collision mode and dedicated mode for SR users. During the operation, the cable isolation layer near the high voltage connector of the DC separator is often melted, which is probably caused by the HOMs power. In order to analyse the power quantitatively and absorb it more effectively, we measured the longitudinal $\mathrm{R} / \mathrm{Q}$ and $\mathrm{Q}$ value of the HOMs, and calculated the HOMs power dissipated in the separator and the connector.

\section{MEASUREMENT OF THE HOMS}

The DC separator is something like a coaxial-cavity, shown in Figure 1.

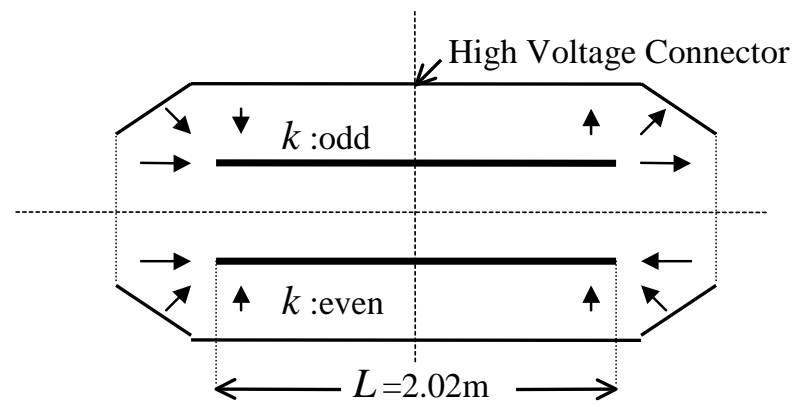

Figure 1. The schematic diagram of the DC separator

The main modes excited by the beam are TEM ones, and their resonant frequencies are:

$$
f_{m} \approx m \cdot \frac{c}{2 L}
$$

where $m=1,2,3,4,5 \ldots, c$ is the speed of the light, $L$ is the length of the inner plate of the separator.
From the characteristics of TEM modes, we know[1]:

- If $m$ is odd, the longitudinal component of the electric field is of the same direction, and the field is weak in the middle of the separator where the high voltage cable is connected to the separator. These are shown in the up side of Figure 1.

- If $m$ is even, the longitudinal component of the electric field is of the opposite direction, and the field is strong in the middle, shown in the low side of Figure 1.

Substituting $L=2.02 \mathrm{~m}$ into equation (1), the resonant frequencies of the HOMs may be got. For example, if $m=1$, then $f_{1}=74.3 \mathrm{MHz}$. This agrees roughly with the measurement value, $f_{1}=71.7 \mathrm{MHz}$. To other $m$, the comparison result is the same. Figure 2 shows the transparent spectrum of the modes. (Those whose frequencies are higher than $750 \mathrm{MHz}$ are omitted, since their shunt impedance is very small)

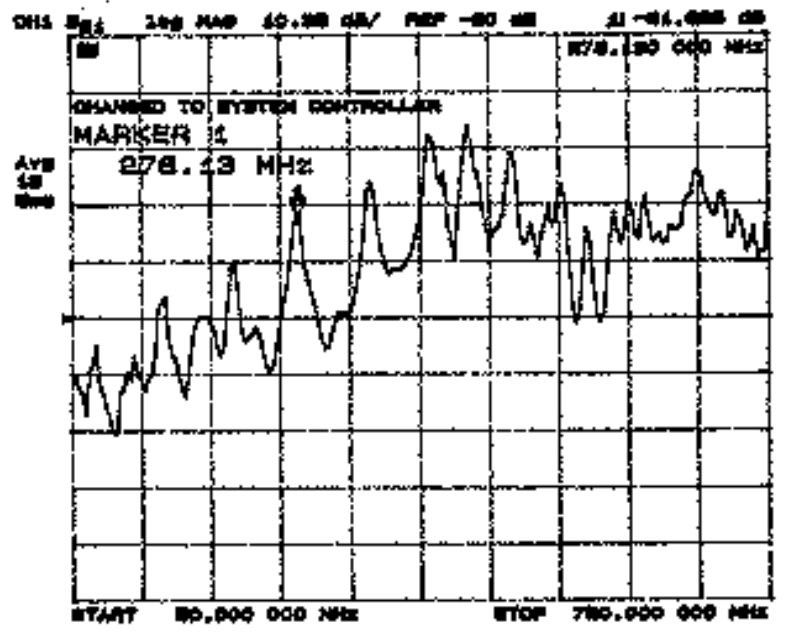

Figure 2 The transparent spectrum of the HOM modes

The longitudinal shunt impedance of the HOMs is measured using the perturbation method. The main instrument of the measuring system is a HP7573C network analyser, controlled by a PC computer. The perturbing object is like a cage, made of some metallic needles glued to the plastic foils at the two ends. The factor of the cage is scaled from a standard cavity. Table 1 shows the measurement results.

\#Email:daijp@bepc4.ihep.ac.cn 
Table $1 \mathrm{HOMs}$ parameters of the DC separator

\begin{tabular}{|c|c|c|c|c|c|}
\hline$m$ & $\begin{array}{c}f_{m} \\
(\mathbf{M H z})\end{array}$ & $\begin{array}{c}R_{m} / Q_{m} \\
(\Omega)\end{array}$ & $Q_{0}$ & $Q_{L}^{\prime}$ & $Q_{L} *$ \\
\hline 1 & 71.73 & 10.5 & 2280 & 2220 & 2163 \\
\hline 2 & 144.86 & 6.5 & 2980 & 745 & 426 \\
\hline 3 & 214.74 & 1.9 & 3700 & 3660 & 3621 \\
\hline 4 & 281.03 & 8.1 & 3238 & 160 & 82 \\
\hline 5 & 354.51 & 0.9 & 3630 & 3586 & 3543 \\
\hline 6 & 428.18 & 6.7 & 2925 & 95 & 48 \\
\hline 7 & 490.57 & 2.7 & 2640 & 2625 & 2610 \\
\hline 8 & 552.5 & 30.4 & 175 & 60 & 30 \\
\hline 9 & 623.51 & 22.5 & 970 & 956 & 942 \\
\hline
\end{tabular}

* The operating separator is connected with two high voltage cables. We measured the loaded $\mathrm{Q}$ merit $\left(Q_{L}^{\prime}\right)$ of the separator with only one cable, and calculated $Q_{L}$ of the separator with two cables.

From table 1 we find that the cables affect the $\mathrm{Q}$ value of the even mode seriously. The reason is the peak of the field of the even mode locates in the middle where the cables are connected

\section{CALCULATION OF HOMS POWER}

Using the cavity impedance and beam spectrum to characterise the interaction between the cavity and the beam in storage rings, a general equation to calculate the HOMs power in the cavity excited by the beam was developed[2]. As a special case, when the storage ring is filled symmetrically with particle bunches, the equation can be simplified[3]. In this paper, we use a computer to simulate the voltage induced in the DC separator by the beams and calculate the HOMs power.

\subsection{Model of the code}

B Gaussian bunches are assumed to be distributed unsymmetrically in storage ring. From the shortest time interval between two bunches in the beam one can find an integer $\mathrm{N}$, the smallest harmonic number of buckets. The buckets are fractionally filled by B bunches, each bunch has the same particle distribution form but could have different number of particles, which is controlled by the normalised filling factor $\alpha_{n}, n=0,1,2, \ldots N-1, \alpha_{k}$ can get the value from zero to one according to the particle numbers in the $n$ bunch. $\alpha_{n}=0$ when the bucket $n$ is empty; $\alpha_{n}=1$ when the bucket has the most particles, whose charge is $q$.

Ignoring the longitudinal oscillation of the bunch and supposing that $J$ turns later after the bunch is injected into the ring, , the voltage $\left(V_{m}(t)\right)$ of the mode at $\omega_{m}$ induced by the beam is saturated and does not change with the turn any more, then:

$$
V_{m}(t)=q H_{m} \cdot\left\{\begin{array}{l}
\sum_{j=1}^{J-1} \sum_{n=1}^{N} \alpha_{n} e^{-\frac{\omega_{m}}{Q_{m}} T_{n j}} \cdot e^{j \omega_{m} T_{n j}} \\
+\sum_{n=1}^{K} \alpha_{n} e^{-\frac{\omega_{m}}{Q_{m}}\left(t-t_{n}\right)} \cdot e^{j \omega_{m}\left(t-t_{n}\right)}
\end{array}\right\}
$$

where

$$
H_{m}=\frac{\omega_{m} R_{m}}{Q_{m}} \cdot e^{-\frac{\left(\omega_{m} \sigma_{l}\right)^{2}}{2}}
$$

$\omega_{m}, R_{m}$ and $Q_{m}$ are the angle frequency, longitudinal impedance and the loaded Q merit of the mode. $\sigma_{l}$ is the bunch length. And,

$$
T_{n j}=t-t_{n}+(J-j) T_{0}
$$

$t_{n}$ is the time of the bunch $n$ traverse the centre of the cavity at turn $j, T_{0}$ is the bunch revolution time in the ring. The bunch $K$ is determined by $t-t_{K} \geq 0$.

Equation (2) is suitable to the case of single beam. If there are two beams in the ring, we need to consider the voltage induced by the other beam. According to the charge of the beams and the field pattern of the mode, we may get the voltage induced by the two beams similarly[2].

With the given voltage is calculated, the power of the mode at $\omega_{m}$ is:

$$
P_{m}=\frac{1}{2 T_{0}} \int_{0}^{T_{0}} \frac{\left|V_{m}(t)\right|^{2}}{R_{m}} d t
$$

The total HOMs power $P_{\text {total }}$ is:

$$
P_{\text {total }}=\sum_{m=1}^{M} P_{m}
$$

where $M$ is the number of the HOMs.

\subsection{Comparison with analytical result}

If the storage ring is filled symmetrically with Gaussian bunches of charge $q$, the HOM power $P_{m}$ of the mode at $\omega_{m}$ is[3][4]:

$P_{m}=\frac{1}{2 T_{b}} \int_{0}^{T_{b}} \frac{\left|V_{m}(t)\right|^{2}}{R_{m}} d t=\frac{1}{T_{b}} \cdot K_{m} \cdot q^{2} \cdot F(\tau, \delta)$

where

$$
\begin{gathered}
F(\tau, \delta)=\frac{(1-\exp (-\tau))(1+\exp (-\tau))}{1-2 \cos \delta \exp (-\tau)+\exp (-2 \tau)} \\
\tau=\omega_{m} T_{b} /\left(2 Q_{m}\right) \\
\delta=\omega_{m} T_{b}
\end{gathered}
$$


$T_{b}$ is the time interval between two bunches, $K_{m}$ is the loss parameter of the mode at $\omega_{m}$,

$$
K_{m}=\frac{1}{2} \omega_{m}\left(\frac{R_{m}}{Q_{m}}\right) \cdot \exp \left(-\left(\omega_{m} \sigma_{l}\right)^{2}\right)
$$

Assume BEPC operates in single bunch mode, and the particle number of the bunch is $3.75 \times 10^{11}$, the buch length is $\sigma_{l}=8.5 \mathrm{~cm}$. For the mode at $\omega_{m}=2 \pi \times 552.5=3471.9 \mathrm{MHz}$, substitute $Q_{m}=30$ and $R_{m} / Q_{m}=30.5 \Omega$ into equation (7), we get the power $P_{m}=95 \mathrm{~W}$. The power calculated by the code is $P_{m}=100 \mathrm{~W}$, agrees well with the analytical result.

\subsection{HOMs power of the BEPC DC separator}

As we know, BEPC usually operates in two modes. By their typical parameters, the code may tell us the HOMs power of the DC separator, shown in table 2.

Table 2 The HOMs power of the DC separator

\begin{tabular}{|c|c|c|c|}
\hline $\begin{array}{c}\text { Operating } \\
\text { Modes }\end{array}$ & $\begin{array}{c}\text { Collision } \\
\text { Mode }\end{array}$ & $\begin{array}{c}\text { SR } \\
\text { Mode I }\end{array}$ & $\begin{array}{c}\text { SR } \\
\text { Mode II }\end{array}$ \\
\hline $\begin{array}{c}\text { Beam Energy } \\
E(\mathrm{GeV})\end{array}$ & 1.548 & 2.2 & 2.2 \\
\hline $\begin{array}{c}\text { Cavity Voltage } \\
V_{c}(\mathrm{MV})\end{array}$ & 1.0 & 0.65 & 0.6 \\
\hline $\begin{array}{c}\text { Bunch Number } \\
B\end{array}$ & 1 & 1 & 60 \\
\hline $\begin{array}{c}\text { Bunch Length } \\
\sigma_{l}(\mathrm{~cm}) * *\end{array}$ & 4.73 & 8.29 & $\sim 4.0$ \\
\hline $\begin{array}{c}\text { Beam Current } \\
I_{t}(\mathrm{~mA})\end{array}$ & 50 & 75 & 110 \\
\hline $\begin{array}{c}\text { Even Mode Power } \\
P_{e}(\mathrm{KW})\end{array}$ & 0.056 & 0.191 & 0.004 \\
\hline $\begin{array}{c}\text { Odd Mode Power } \\
P_{o}(\mathrm{KW})\end{array}$ & 0.023 & 0.095 & 0.001 \\
\hline$*$
\end{tabular}

a) In single bunch case, $\sigma_{l}$ is given by the scaling law of BEPC bunch length

$$
\sigma_{l}=0.404 \times\left(\frac{I_{b}(m A) \alpha_{p}}{E(G e V) v_{s}^{2}}\right)^{\frac{1}{2.80}}
$$

where $I_{b}$ is the current of the single bunch, $\alpha_{p}$ is momentum compression factor, $v_{s}$ is the longitudinal tune.

b) In multi-bunch case, since the bunches have different charges, $\sigma_{l}$ is an average value.
From table 1, we know that the power of the odd mode dissipates mainly in the seperator itself, while the power of the even modes dissipateds mainly in the high voltage cable and the connector which will cause the isolation layer to melt.

\subsection{Analysis of the result}

Since there are some differences between the operating separator and the measured one, and the measurement errors are unavoidable, we need to consider the error between the calculated power dissipated in the measured separator and the actual one in the operating separator. By changing the parameters: the frequency, the $\mathrm{Q}$ merit and $R_{m} / Q_{m}$ in the code, we find that:

- To the odd modes, the power error caused by the frequency error may be very large. For example, to the mode $m=7$, if the frequency is $f_{7}=491.37 \mathrm{MHz}$ instead of $f_{7}=490.57 \mathrm{MHz}$, then the power will be $58 \mathrm{~W}$ (in the mode SR I), not $4 \mathrm{~W}$. However, to the even modes, which we are interested in, is very small because of the very low $Q$ merit.

- The power error caused by the $\mathrm{Q}$ merit error is small

- The power is almost proportional to $R_{m} / Q_{m}$, so the power error will not be very large since the measured $R_{m} / Q_{m}$ is not very large.

\section{ACKNOWLEDGEMENT}

We would like to express our great appreciation to our colleagues: Y.D. Hao, Z.Y. HAO and S.P. Li for their help in the measurement of the separator.

\section{REFERENCES}

[1]F. Zhou, "Research of impedance theory and its application", Doctor Thesis, IHEP , (1997)

[2]Z.T. Zhao , "The HOMs power generated by unequally spaced and populated bunches in storage ring", High Energy Physics and Nuclear Physics, (to be pulished)

[3]Haebel E., "RF design -higher order modes", Lecture Note in Physics No.425, W.Beiglbock, et at, Eds, Spinger-Verlag, Berlin Heidelberg, (1992)

[4]Wilson P., "High energy electron linacs: application to storage ring RF systems and linac collider", SLAC-PUB-2884, (Feb. 1982) 